\title{
A case of Strongyloides hyperinfection associated with tuberculosis
}

\author{
Yoshiaki Iwashita*, Kei Suzuki, Asami Masui, Eiji Kawamoto, Kazuto Yokoyama, Akitaka Yamamoto, Yukinari Omori, \\ Ken Ishikura, Tsuyoshi Hatada, Masaki Fujioka, Taichi Takeda and Hiroshi Imai
}

\begin{abstract}
Strongyloidiasis is a parasitic infection that occurs in tropical regions. Hyperinfection, which is an accelerated autoinfection, is often associated with an immunosuppressive state, such as HTLV-1 infection or steroid use. Immunosuppression can also lead to reactivation of tuberculosis infection. These infections may have interacted as a result of impaired cellular immunity. A 28-year-old Nepali male was referred to our hospital for slight abdominal pain and high fever. An abdominal CT scan showed ascites and intestinal swelling. He was admitted with suspected gastroenteritis. Results of stool microscopy on the third day of hospitalization revealed abundant strongylid larvae. We diagnosed a Strongyloides hyperinfection and prescribed ivermectin. Although the numbers of strongylid organisms in the patient's stool soon diminished, his temperature remained high. After receiving a second dose of ivermectin on day 17, he was transferred to a nearby hospital for observation, where he was noted to have massive pleural effusion. He returned to our hospital where his pleural effusion was found to be positive for adenosine deaminase (ADA), and he was diagnosed with a tuberculosis infection. Strongyloides hyperinfection can occur in a non-endemic region. It can be associated with tuberculosis infection possibly due to impaired cellular immunity. It is important to consider other possible infections when treating a patient with an infection associated with impaired cellular immunity.
\end{abstract}

Keywords: Strongyloidiasis, Hyperinfection, Ivermectin, Tuberculosis, Cellular immunity

\section{Background}

Strongyloidiasis is a parasitic infection rarely seen in mainland Japan. Hyperinfection of this organism sometimes leads to fatal outcome because hyperinfections often occur in patients whose cellular immunity is impaired. Here, we present a case involving a 28-year-old Nepali male suffering from a hyperinfection of Strongyloides whom we successfully treated with ivermectin. After the strongyloidiasis was treated, the patient was found to have tuberculosis. This case may suggest a relationship between strongyloidiasis and tuberculosis attributable to impaired cellular immunity.

\section{Case presentation}

A 28-year-old Nepali male was referred to our hospital with suspected gastroenteritis. He had a 3-week history of high fever (around $38^{\circ} \mathrm{C}-39^{\circ} \mathrm{C}$ ), appetite loss, and general

\footnotetext{
*Correspondence: iwaci1ta@clin.medic.mie-u.ac.jp
Emergency and Critical Care Center, Mie University hospital, 2-147 Edobashi,

* Correspondence: iwaci1 ta@clin.medic.mie-u.ac.jp
Emergency and Critical Care Center, Mie University hospital, 2-147 Edobashi, Tsu, Mie, Japan
}

(c) 2013 Suzuki et al.; licensee BioMed Central Ltd. This is an open access article distributed under the terms of the Creative Commons Attribution License (http://creativecommons.org/licenses/by/2.0), which permits unrestricted use, distribution, and reproduction in any medium, provided the original work is properly cited. found, on abdominal $C T$, to have ascites and duodenal swelling without free air. On admission to our hospital, he complained of general fatigue and slight abdominal pain but was not experiencing diarrhea. He had been in Japan for 4 years and had not been back to Nepal for the last 2 years. He was living with four other Nepali males who were not experiencing similar physical problems. He denied being males who sex with males. He was a Hindu and ate Japanese food bought in a nearby supermarket. He had no remarkable past medical history and was not taking any medications. He works so hard on a supermarket in Japan and he could not sleep well. He also feels loss of appetite. He had been repeated low-grade fever around $37^{\circ} \mathrm{C}$ and general fatigue before coming to our hospital.

In general, the patient did not look sick. His height was $170 \mathrm{~cm}$, weight was $46.5 \mathrm{~kg}$, and BMI was 16.1 . His blood pressure was 90/64, pulse rate 109/min, and body temperature $36.2^{\circ} \mathrm{C}$. His eyes were not anemic or icteric, and chest sounds were clear. His abdomen was soft and 
flat, although there was slight tenderness in the left lower quadrant.

Laboratory data on admission showed a slightly elevated

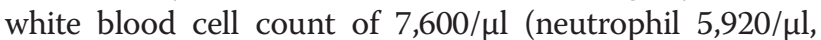
lymphocyte $880 / \mu \mathrm{l}$, monocyte $790 / \mu \mathrm{l}$, eosinophil $0 / \mu \mathrm{l}$, and

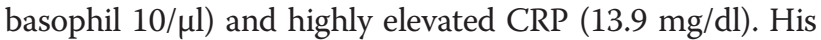
albumin was low at $3.0 \mathrm{~g} / \mathrm{dl}$. A chest radiograph was clear, and there were no signs of pneumonia. An abdominal computed tomography (CT) scan on admission showed ascites with swollen intestines but no free air (Figure 1).

We hospitalized the patient for observation. His temperature rose to $40^{\circ} \mathrm{C}$, and laboratory data on day 2 showed highly elevated inflammatory markers. Microbiological studies including sputum, urine, and blood cultures were all negative. He was constipated until day 3 . His stool on day 3 looked normal; it was not watery and contained no blood. Stool microscopy revealed numerous strongylid larvae (Figure 2). We diagnosed a Strongyloides hyperinfection. Serum human T-cell leukemia virus type 1 (HTLV-1) and human immunodeficiency virus (HIV) tests were negative, and CD4 counts were not decreased. We prescribed $12 \mathrm{mg}$ of ivermectin. Although his general condition improved within $24 \mathrm{~h}$ after treatment in that he was eating well and had no diarrhea or abdominal pain, his temperature continued to exceed $39^{\circ} \mathrm{C}$. The course of his inflammatory laboratory data and temperature are shown in Figure 3. On day 7, his stool sample was negative for strongylid larvae. An abdominal CT scan on day 6 revealed massive ascites, but the culture of the ascites on day 9 was negative for strongylid larvae. He did not complain of dyspnea throughout his hospitalization. A second 12-mg dose of ivermectin was administered on day 17.

After the second dose of ivermectin, the patient still had a low-grade fever of $37^{\circ} \mathrm{C}-38^{\circ} \mathrm{C}$. His laboratory data were within the normal limit except for relatively low albumin
(2.6 g/dl) and still low lymphocyte count $(470 / \mu \mathrm{l})$. He looks lethargy. We thought this is due to long-term hospitalization; thus, we transferred him to another hospital for further rehabilitation. However, he returned to our hospital 4 days later for pleural effusion of unknown etiology. He had massive pleural effusion in his left chest, and his ascites had not diminished. Thoracentesis was performed on admission, and the pleural fluid was positive for adenosine deaminase (ADA) as $128 \mathrm{IU} / \mathrm{l}$. Ascites were also positive for ADS as $156.6 \mathrm{IU} / \mathrm{l}$. QuantiFERON was positive, and mycobacterial culture of gastric secretions revealed Mycobacterium tuberculosis. Sputum culture and Gaffky staining were negative. Repeated stool microscopy was negative for Strongyloides. The patient transferred to another hospital for tuberculosis treatment.

We encountered a case of Strongyloides hyperinfection, which is rarely seen in Japan. This patient also developed a tuberculosis infection after successful treatment of his strongyloidiasis. Although the relationship between these two infections is not apparent, there may be an association because of the unique characteristics of these infections.

Strongyloidiasis is endemic in tropical and subtropical regions. In Japan, the Okinawa prefecture, which is in the southern islands, is the only endemic region [1]. Because our patient had never been to the Okinawa prefecture, we believe he became infected in Nepal. Strongyloides can cause acute, chronic, hyper, and disseminated infection. Strongyloides hyperinfection occurs due to the unique life cycle of the Strongyloides stercoralis. Their eggs are embedded in the intestinal mucosal epithelium, and noninfectious rhabditiform larvae hatch into the intestinal lumen. The rhabditiform larvae become free-living adults when secreted in feces into the soil. They can develop to infectious filariform larvae, at which point they can penetrate human skin and invade the intestine via the
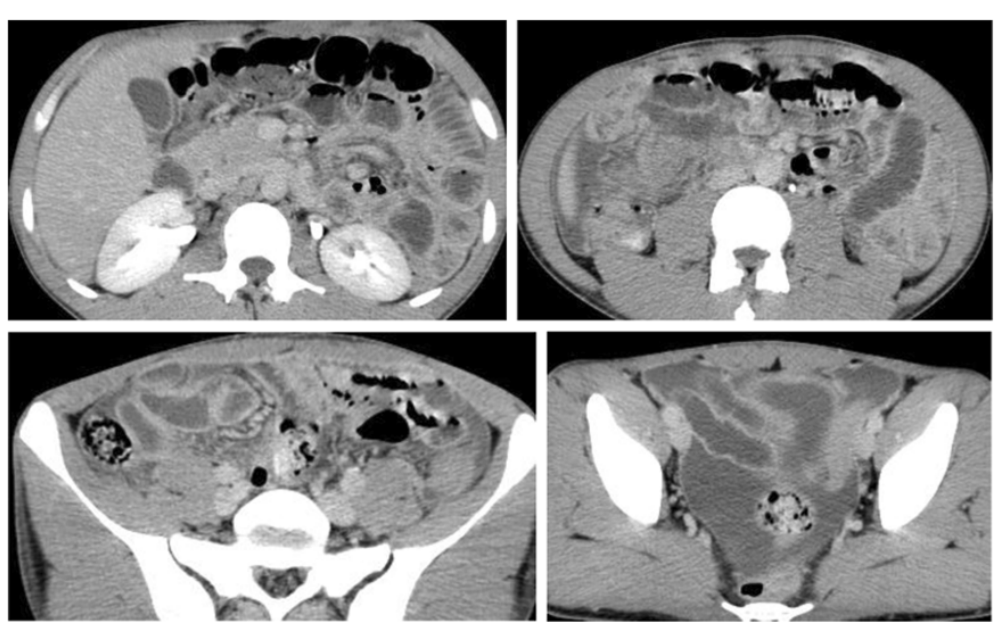

Figure 1 Abdominal CT scan on admission. Ascites and intestinal swelling can be seen. 


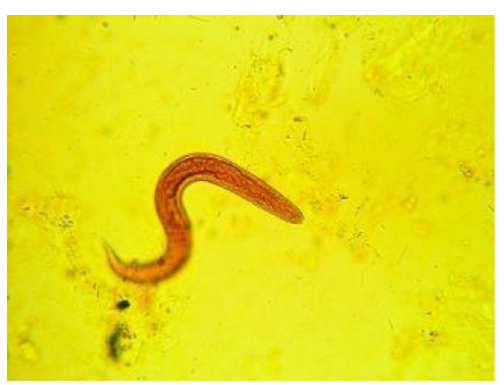

$\times 100$

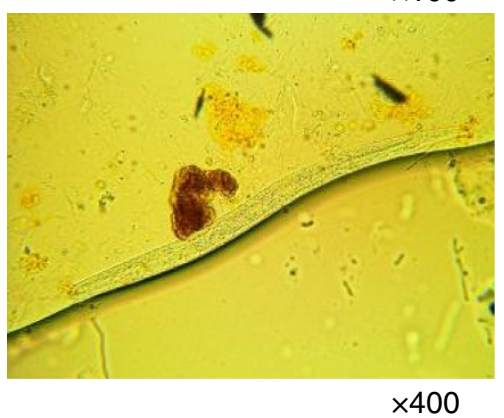

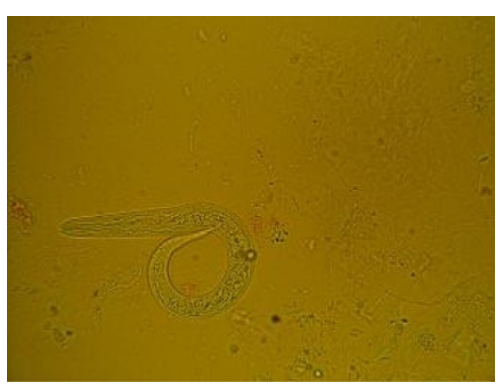

$\times 100$ bloodstream [2]. Strongyloides hyperinfection is an accelerated autoinfection in which increasing numbers of infectious filariform larvae are produced. Such hyperinfections can occur when the host becomes immunosuppressed, such as with steroid use or HTLV-1 infection [2]. These infections can occur even 57 years after chronic infection [3]. In chronic infection, more than $50 \%$ of patients are asymptomatic [4]. Our patient had no symptoms and had not been to an endemic area for 2 years; therefore, we believe this patient had been chronically infected since he was in Nepal and became hyperinfected in Japan. Although this patient does not have history of steroid use or HTLV-1 infection. The patient had worked so hard that he could not eat well. Malnutrition induced decreased number of lymphocyte and subsequently hyperinfected with Strongyloides.

Tuberculosis is a re-emerging infection in Japan and is endemic in Nepal. It is an infection that can be re-

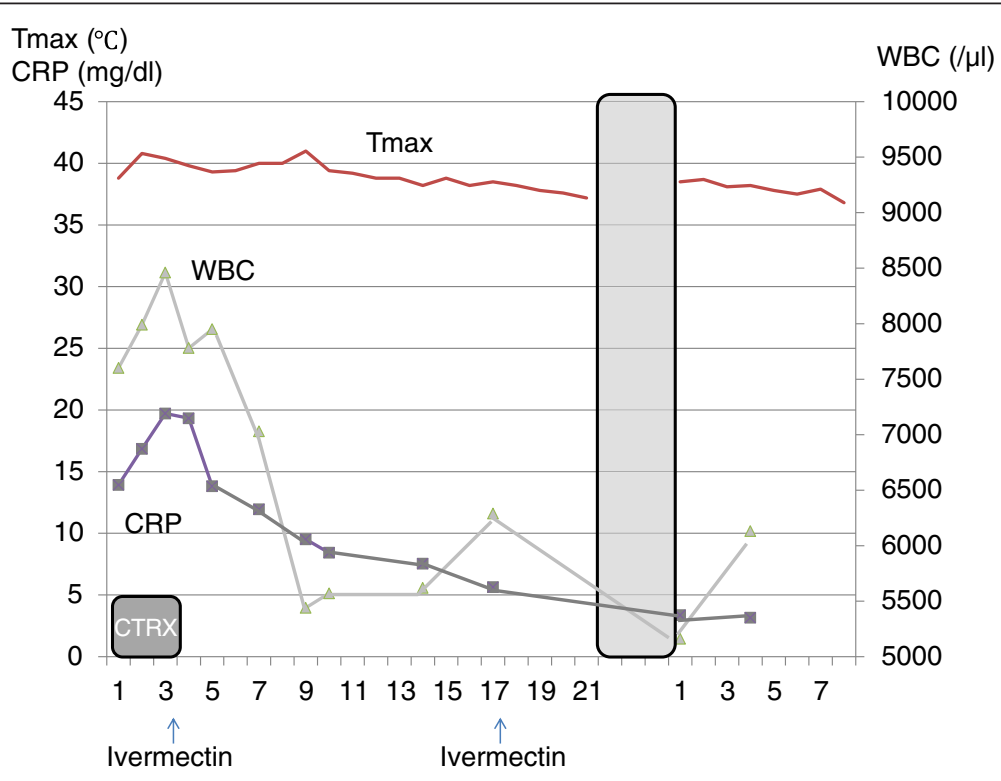

Figure 3 Hematology and clinical chemistry data. Although the patient's markers of inflammation decreased after ivermectin administration, his temperature remained high. 
activated when the host becomes immunosuppressed. There is one previous report in the literature of a coinfection with strongyloidiasis and tuberculosis [5]. The authors concluded that their patient developed tuberculosis because of Strongyloides hyperinfection. However, the possibility that the patient developed strongyloidiasis hyperinfection during the course of the tuberculosis infection is not discussed. Similarly, in our patient, it is not clear whether the strongyloidiasis stimulated the tuberculosis reactivation or vice versa. However, it is clear that both Strongyloides infection and tuberculosis infection are associated with impaired cellular immunity [6].

Our patient's lymphocyte count was low, indicating that he was at risk of infection with both strongyloidiasis and tuberculosis. We estimated that malnutrition due to poor diet caused decreased lymphocyte. The decreased lymphocyte caused Strongyloides hyperinfection and tuberculosis. We believe Strongyloides hyperinfection occurred earlier and subsequently tuberculosis, because there were no signs and symptoms of tuberculosis infection on admission. Regardless, patients with either strongyloidiasis or tuberculosis should be examined carefully for other infections, particularly when the patient has impaired cellular immunity.

\section{Conclusion}

We encountered a unique case in Japan of Strongyloides infection in a patient who subsequently was diagnosed with tuberculosis. These infections can therefore present even in non-endemic regions. It is important to consider additional infections when treating a patient with an infection caused by impairment of cellular immunity.

\section{Consent}

Written informed consent was obtained from the patient for publication of this case report and any accompanying images. A copy of the written consent is available for review by the Editor-in-Chief of this journal.

\section{Competing interests}

The authors declare that they have no competing interests.

\section{Authors' contributions}

YI drafted the manuscript. KS contributed to make a diagnosis and treatment strategy of this patient. AM, EK, KY, AY, YO, KI, TH, MF and TT contributed to treat this patient. $\mathrm{HI}$ conceived writing this report and coordinated to draft the manuscript. All authors read and approved the final manuscript.

Received: 19 July 2013 Accepted: 7 October 2013

Published: 26 November 2013

\section{References}

1. Hirata T, Nakamura H, Kinjo N, Hokama A, Kinjo F, Yamane N, Fujita J: Prevalence of Blastocystis hominis and Strongyloides stercoralis infection in Okinawa, Japan. Parasitol Res 2007, 101:1717-1719.

2. Mandell GL, Bennett JE, Dolin R: Mandell: Mandell, Douglas, and Bennett's principles and practice of infectious diseases. 7th edition. Philadelphia, PA: Churchill Livingstone.
3. Gill GV, Beeching NJ, Khoo S, Bailey JW, Partridge S, Blundell JW, Luksza AR: A British Second World War veteran with disseminated strongyloidiasis. Trans R Soc Trop Med Hyg 2004, 98:382-386.

4. Grove Dl: Human strongyloidiasis. Adv Parasitol 1996, 38:251-309.

5. Dwarakanath AD, Welton M, Ellis CJ, Allan RN: Interrelation of strongyloidiasis and tuberculosis. Gut 1994, 35:1001-1003.

6. Abdelrahman MZ, Zeehaida M, Rahmah N, Norsyahida A, Madihah B, Azlan H, Nazlee WZ: Fatal septicemic shock associated with Strongyloides stercoralis infection in a patient with angioimmunoblastic T-cell lymphoma: a case report and literature review. Parasitol Int 2012, 61:508-511.

doi:10.1186/2052-0492-1-7

Cite this article as: Iwashita et al:: A case of Strongyloides hyperinfection associated with tuberculosis. Journal of Intensive Care 2013 1:7.

\section{Submit your next manuscript to BioMed Central and take full advantage of:}

- Convenient online submission

- Thorough peer review

- No space constraints or color figure charges

- Immediate publication on acceptance

- Inclusion in PubMed, CAS, Scopus and Google Scholar

- Research which is freely available for redistribution 